\title{
O CONHECIMENTO DAS ADOLESCENTES SOBRE QUESTÕES RELACIONADAS AO SEXO
}

\author{
Kelencristina T. Romero, Élide Helena G. R. Medeiros, Maria Sylvia S. Vitalle*, Jamal Wehba
}

Trabalho realizado no Centro de Atendimento e Apoio ao Adolescente do Departamento de Pediatria da Universidade Federal de São Paulo (Unifesp/EPM).

\author{
*Correspondência: \\ Centro de Atendimento e \\ Apoio ao Adolescente da \\ Unifesp/EPM \\ Rua Botucatu, 715 \\ São Paulo/SP \\ Cep: 04023-062 \\ vitalle.dped@epm.br
}

\begin{abstract}
RESUMO
Oвjetivo. Avaliar o conhecimento sobre sexualidade, métodos contraceptivos e doenças sexualmente transmissíveis (DST) entre adolescentes do sexo feminino, das zonas rural e urbana, de uma escola pública.

Métodos. Estudo transversal, realizado com 506 meninas, com idades entre 10 e 16 anos, da Escola Dr. Roberto Feijó, em Guararema, SP. Utilizou-se questionário semi-estruturado, contendo perguntas gerais sobre sexualidade e métodos de prevenção de gravidez e DST. O teste do Qui-quadrado foi usado para verificar a associação entre as variáveis.

Resultados. A média de idade da população adolescente da escola proveniente da área rural foi I 3 anos e II meses e da área urbana foi 13 anos e 7 meses, não havendo diferença estatística entre as médias. Trinta e um por cento eram provenientes da zona rural e $69 \%$ da urbana. As jovens da zona rural buscaram mais informações sobre a sexualidade $(81,2 \%)$, comparadas com as da zona urbana $(72,2 \%)(p<0,0568)$, e a principal fonte de informação foram os pais nas duas regiôes. A camisinha foi o método contraceptivo mais conhecido pelas adolescentes em ambas as áreas, $44 \%$ a conheciam na zona rural e $45 \%$ na urbana $(p=0,0022)$. A Aids é a DST mais conhecida nos dois grupos; $43 \%$ das jovens da zona rural e $39 \%$ da zona urbana referiram conhecer a doença $(p=0,7843)$.
\end{abstract}

Conclusão. A maioria das adolescentes buscou informações sobre sexualidade, mas os conhecimentos sobre doenças sexualmente transmissíveis e contracepção são inadequados.

Unitermos: Adolescente. Sexualidade. Anticoncepção. Doenças Sexualmente Transmissíveis. Comportamento.

\section{INTRODUÇÃo}

No Brasil, em torno de $19 \%$ da população geral constituem-se de adolescentes, segundo o censo de 2000, isto é, 34 milhões de jovens'. Neste período, ocorrem mudanças biopsicossociais, tais como maturação dos caracteres sexuais secundários; independência socioeconômica e emocional dos pais; elaboração da identidade pessoal e sexual; aquisiçã̃o do pensamento abstrato; exercício da sexualidade, intimidade e afetividade ${ }^{2,3}$. Frente a estas transformações, observa-se que o desenvolver da sexualidade está intimamente ligado ao desenvolvimento integral do indivíduo.

Observa-se, na atualidade, que a atividade sexual se inicia cada vez mais precocemente. Estudos nos anos 90 mostravam que a média de idade da primeira relação sexual do sexo feminino era de 16 anos, e que $70 \%$ das adolescentes com 19 anos tiveram pelo menos uma relação sexual ${ }^{4,5}$. Em 2000, no Brasil, a média de idade da primeira relação sexual em meninas foi de 15 anos 6 .

Existem vários fatores importantes que podem influenciar de modo adverso a saúde sexual e reprodutiva da adolescente, comprometendo o seu processo natural de crescimento e desenvolvimento, entre os quais pode-se citar a gravidez precoce, muitas vezes indesejada, as doenças sexualmente transmissíveis (DST), os acidentes, a violência, os maus tratos, o uso de drogas e a evasão escolar ${ }^{7,8}$.

A sexualidade é, portanto, elemento significante na formação da identidade da adolescente, manifestada por múltiplas identificações, como da imagem corporal, da descoberta do outro como objeto de amor ou desejo e da descoberta de si e das relações com os familiares, grupos e profissionais?. Embora o exerćício da sexualidade seja considerado uma conduta simples e cotidiana, é muito complexa e permeia aspectos cognitivos que vão desde os mais primitivos (sensoriais) até esquemas de representação mais complexos, que envolvem a linguagem corporal, facial e outros sistemas de sinais. Há, ainda, os aspectos culturais, extremamente relevantes, também imbricados na formação e no exercício da sexualidade humana. Apesar da importância do tema para a formação integral da adolescente, existem poucos estudos no Brasil a esse respeito e, na prática diária da profissão, os pediatras estão pouco afeitos a tratar da questão da sexualidade. Por outro lado, nesta época aflora a temática da sexualidade ${ }^{10}$.

Assim, o objetivo deste trabalho foi avaliar o conhecimento das adolescentes sobre sexualidade, métodos contraceptivos e doenças sexualmente transmissíveis em uma escola pública, da cidade de Guararema, São Paulo, para futuramente se construir um programa de saúde integral ao adolescente na rede escolar na cidade.

\section{MÉTOdos}

Em Guararema, SP, a população entre 10 e 19 anos representa 19,8\% do total de habitantes', o que equivale a 4348 indivíduos. Destes, encontravam-se matriculados, no ano de 200I, 3345 alunos nas escolas públicas da cidade, sendo que $48,7 \%$ e $51,3 \%$, respectivamente no ensino fundamental e médio, eram do sexo feminino".

Este é um estudo observacional, transversal, prospectivo, realizado no período de $1^{\circ}$ de junho a 30 de novembro de 200I, na Escola Estadual Dr. Roberto Feijó, com adolescentes do sexo feminino 
matriculadas entre a quinta série do ensino fundamental e o primeiro ano do ensino médio. A seleção da escola foi feita por conveniência, condicionada à permissão dos dirigentes. Como era possível avaliar a totalidade dos estudantes nesta escola, não foi necessário trabalhar com técnicas de amostragem.

Este trabalho foi aprovado pelo Comitê de Ética em Pesquisa da Universidade Federal de São Paulo. Foi obtido dos pais ou responsáveis e da adolescente o prévio consentimento por escrito, sendo a não autorização ou a adolescente não querer participar da pesquisa o único critério de exclusão. $O$ critério de inclusão consistia em ser aluna regularmente matriculada na escola no ano letivo e ser adolescente, isto é, estar na faixa etária dos 10 aos 20 anos incompletos.

Utilizou-se um questionário semi-estruturado, autopreenchível, anônimo, contendo perguntas gerais sobre sexualidade, métodos contraceptivos, gravidez, doenças sexualmente transmissíveis, Aids e namoro, desenvolvido pelos autores a partir de um modelo de experiência anterior, usando em parte o modelo proposto por Rodrigues $\mathrm{Jr}^{12}{ }^{12}$ no tocante à sexualidade. Foi realizado um teste piloto de questionários, em adolescentes, para corrigir imperfeições. As seguintes variáveis foram avaliadas e categorizadas: busca de informações sobre sexualidade (sim, não), com quem conversa sobre este assunto (questão aberta), quais métodos preventivos conhece para gestação e DST (questão aberta), quais DST conhece (Aids, gonorréia, condiloma ou Papilomavírus, sífilis, linfogranuloma venéreo, tricomoníase, candidíase, herpes genital, cancro mole, hepatite B), as formas de contágio das DST (sexo oral, mãe para filho no parto, sexo anal, amamentação, sexo genital, banheiro público, beijo na boca, drogas injetáveis, abraço, picadas de insetos, roupas íntimas, piscinas, banheiras e aperto de mãos) e as formas de contágio da Aids (sexo oral, mãe para filho no parto, sexo anal, amamentação, sexo genital, banheiro público, beijo na boca, drogas injetáveis, abraço, picadas de insetos, roupas íntimas, piscinas, banheiras, aperto de mãos, transfusão de sangue contaminado com uso de seringas estéreis, toque em superfícies contaminadas).

Os questionários foram respondidos em sala de aula, após explanação do pesquisador sobre o objetivo e a natureza da pesquisa, sendo mantidos o anonimato e a confidencialidade.

Para verificar a associação entre as variáveis, aplicou-se o teste do Qui-quadrado, fixando-se em 0,05 o nível de rejeição da hipótese de nulidade e assinalando-se com asterisco os valores significantes.

\section{Resultados}

A população deste estudo constitui-se de 506 meninas, com idades entre 10 e 16 anos, sendo que 10,47\% das adolescentes não aceitaram participar, restando 453 voluntárias. Destas, I 40 (31\%) moravam na zona rural e 313 (69\%) na zona urbana, e a idade média das jovens da área rural foi de 13 anos e I I meses e da área urbana foi de 13 anos e 7 meses, não havendo diferença estatística entre elas.

A Tabela I refere-se à busca de informações sobre sexualidade, observando-se que a porcentagem de adolescentes da zona rural que procuravam informações $(81,2 \%)$ foi significativamente maior do que a da zona urbana $(72,2 \%)$, com $p<0,00568$.

A Tabela 2 mostra as principais fontes de informação sobre sexualidade para as adolescentes; nas zonas rural e urbana, mais de $50 \%$ das jovens conversavam com os pais e as amigas para obterem

\begin{tabular}{|c|c|c|c|c|}
\hline \multirow[t]{2}{*}{ Zona de residência } & \multicolumn{4}{|c|}{ Buscam informações } \\
\hline & Sim & Não & Total & $\%$ Sim \\
\hline Rural & 112 & 26 & 140 & $81,2^{*}$ \\
\hline Urbana & 226 & 87 & 309 & 72,2 \\
\hline Total & 338 & 113 & 450 & 74,9 \\
\hline
\end{tabular}

Teste do Qui-quadrado $=3,63 * ; p<0,0568$

p: nível de significância do teste do Qui-quadrado

$\S$ Em três casos, não havia informação para esta variável

conhecimentos sobre o assunto, não havendo diferenças estatísticas entres as zonas.

Estão relacionados, na Tabela 3, os métodos preventivos que as adolescentes referiram conhecer para evitar as DST e a gravidez, não havendo diferenças entre as duas zonas de residência, exceto no tocante ao certificar-se de que o parceiro não tem doença sexualmente transmissível, como método de prevenção de DST $(p<0,000 \mathrm{I})$, e ao uso de preservativo como prevenção de gestação $(p=0,0022)$.

Não foi observada diferença estatisticamente significante quanto ao conhecimento sobre DST entre a população das zonas rural e urbana $(p=0,7843)$. Na zona rural, 128 adolescentes referiram conhecer as moléstias e, na zona urbana, 282 delas referiram também ter conhecimento (dados não mostrados).

A camisinha foi o método contraceptivo mais conhecido pelas adolescentes em ambas as áreas, sendo conhecido por 44\% das jovens na zona rural e por $45 \%$ na urbana (Tabela 3), sendo que, proporcionalmente, as da zona urbana têm melhor conhecimento $(p=0,0022)$.

Quanto às DST, os dois grupos relataram ser a Aids a doença mais conhecida: 43\% na zona rural e 39\% na urbana ( $p=0,7843)$. Apesar disso, responderam incorretamente sobre a forma de aquisição da doença (24\% na zona rural e $21 \%$ na urbana); sendo a porcentagem de conhecimento das outras afecções no primeiro grupo: hepatite B (19\%), gonorréia (13\%), herpes genital (11\%), sífilis (10\%), candidíase (2\%), condiloma ou Papilomavírus (I,5\%), e no segundo grupo: hepatite B (20\%), gonorréia (I2\%), herpes genital (13\%), sífilis ( I 1\%), candidíase (2\%), cancro mole (2\%) e condiloma ou Papilomavírus (1\%). As adolescentes desconhecem doenças como tricomoníase e linfogranuloma venéreo. $O$ conhecimento de DST em ambas as áreas foi semelhante, não havendo diferença estatística (dados não mostrados).

Interrogou-se, ainda, sobre as formas de contágio das DST e da Aids, porém, não foi possível realizar análise estatística devido ao número de variáveis e ao tamanho da amostra. Para as DST, as respostas mais freqüentes na zona rural foram: drogas injetáveis (17\%), sexo genital ( $16 \%)$, sexo anal (14\%), sexo oral ( $12 \%)$, piscinas, banheiras e banheiros públicos (12\%), mãe para filho ao nascer (8\%), roupas íntimas (7\%), amamentação (5\%) e $8 \%$ das adolescentes acreditavam que DST se contrai por picadas de insetos (3\%), abraços, aperto de mãos ou beijo na boca (2\%). 
Tabela 2 - Fontes de informação sobre sexualidade segundo a zona de residência. Tamanho da amostra (N), percentual e análise estatística. Escola Dr. Roberto Feijó, Guararema, 200I

\begin{tabular}{|c|c|c|c|c|c|c|c|c|}
\hline \multirow[t]{3}{*}{ Fonte de informação } & \multicolumn{6}{|c|}{ Zona de residência } & \multicolumn{2}{|c|}{ Análise estatística } \\
\hline & \multicolumn{2}{|c|}{ Rural } & \multicolumn{2}{|c|}{ Urbana } & \multicolumn{2}{|c|}{ Total } & \multirow[t]{2}{*}{ Qui-quadrado } & \multirow[t]{2}{*}{$p$} \\
\hline & $\mathbf{N}$ & $\%$ & $\mathbf{N}$ & $\%$ & $\mathbf{N}$ & $\%$ & & \\
\hline Mãe/pai & 63 & 38,42 & 153 & 39,44 & 216 & 39,13 & 0,4390 & 0,5076 \\
\hline Amiga & 40 & 24,39 & 107 & 27,58 & 147 & 26,63 & 1,146 & 0,2843 \\
\hline Ninguém & 17 & 10,37 & 35 & 9,02 & 52 & 9,42 & 0,0887 & 0,8911 \\
\hline Irmã & 9 & 5,49 & 19 & 4,9 & 28 & 5,08 & 0,0214 & 0,8837 \\
\hline Profissionais ${ }^{\S !}$ & 3 & 1,83 & 10 & 2,58 & 13 & 2,35 & 0,0993 & 0,7526 \\
\hline Outros ${ }^{\S 2}$ & 12 & 7 & 30 & 8 & 42 & 8 & 0,0283 & 0,8663 \\
\hline Total $^{\mid 3}$ & 164 & 29,71 & 388 & 70,29 & 552 & 100 & & \\
\hline
\end{tabular}

p: nível de significância do teste do Qui-quadrado

$\S 1$ : médico/professor

$\S 2$ : pessoas mais experientes, namorado e sem respostas

§3: o número total ultrapassou o tamanho da amostra, pois houve mais de uma resposta para cada variável

Tabela 3 - Conhecimento sobre os métodos de sexo seguro (prevenção de DST e gestação) segundo a zona de residência.

Tamanho da amostra (N), percentual e análise estatística. Escola Dr. Roberto Feijó, Guararema, 200I

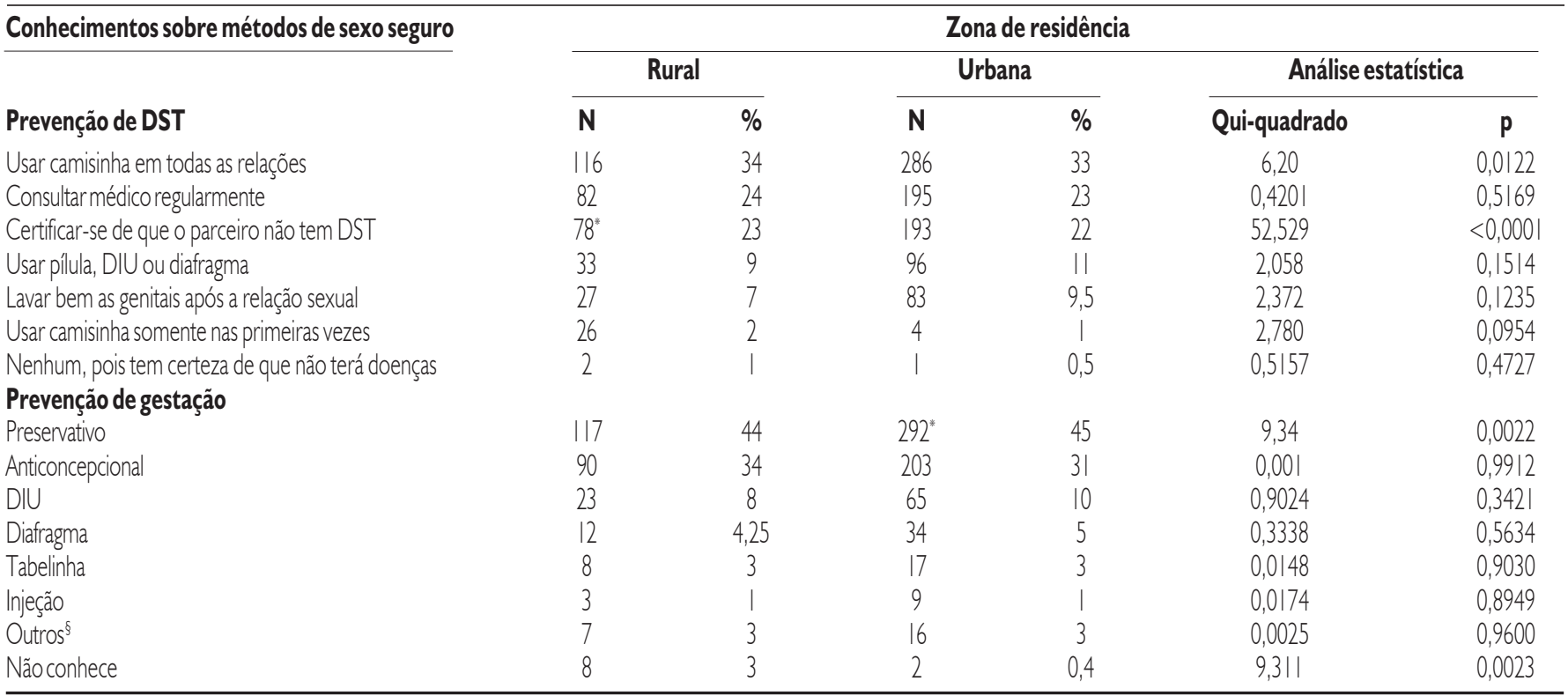

§: Camisinha feminina, laqueadura ou esterilização, coito interrompido, pomada, não transar ou não namorar, muco

p: nível descritivo do teste Qui-quadrado

As respostas foram semelhantes na zona urbana, sendo a de maior freqüência o sexo genital (20\%), seguido de drogas injetáveis (16\%), sexo anal (15\%), sexo oral (14\%), mãe para filho ao nascer (10\%), roupas íntimas (6\%), amamentação (3\%) e 2,5\% das adolescentes acreditavam que o contágio ocorre por picadas de insetos, abraços e aperto de mãos (0,5\%) ou beijo na boca (2\%).

Com relação à Aids, as adolescentes de área rural referiram que a aquisição da doença ocorria por transfusão de sangue contaminado (17\%), sexo genital (I4\%), drogas injetáveis (I2\%), sexo anal (12\%), uso de seringas estéreis (10\%), sexo oral (8\%), mãe para filho ao nascer (8\%), amamentação (4\%) e um total de I2\% das adolescentes acreditam que se contrai em picadas de insetos, piscinas, banheiras, banheiros públicos ou toque em superfícies contaminadas. As adolescentes de zona urbana citaram transfusão de sangue contaminado (I7\%), sexo genital (I7\%), drogas injetáveis (I 2\%), sexo anal (I I\%), sexo oral (9\%), uso de seringas estéreis (8\%), mãe para filho ao nascer $(8,5 \%)$, amamentação $(3,5 \%)$ e $11 \%$ das adolescentes acreditavam que o contágio ocorresse em picadas de insetos, piscinas, banheiras, banheiros públicos, aperto de mãos ou toque em superfícies contaminadas. 
Vinte e cinco por cento de todas as adolescentes entrevistadas informaram que não sabiam como se contrai DST e Aids.

Frente a estes resultados, analisou-se a porcentagem de respostas corretas sobre o conhecimento da forma de contágio das DST e observou-se acerto de $73 \%$ na zona rural e $77 \%$ na urbana $(p=0,1056)$; e da Aids nas freqüências de $76 \%$ e $79 \%$ $(p=0,1556)$, respectivamente, não havendo diferenças estatisticamente significantes.

\section{Discussão}

Observou-se que $74,9 \%$ das adolescentes buscavam informações sobre sexualidade, principalmente na zona rural (Tabela I); isto pode ser decorrente da desinformação que permeia áreas de baixo nível socieconômico ou do fato de que se voltam para esta questão mais precocemente.

Não há dúvida de que, atualmente, as adolescentes falam mais sobre sexo com os pais. Contudo, as conversas transitam apenas na superficialidade, não há esclarecimento sobre a necessidade de alguns cuidados antes da iniciação sexual e do conhecimento adequado dos métodos contraceptivos. Os amigos freqüentemente também são procurados, mas as conversas começam interessantes e posteriormente acabam na vulgarização, deixando sérias dúvidas sobre a validade do conteúdo e a seriedade do diálogo $\mathrm{O}^{13,14}$.

Neste estudo, os pais foram a principal fonte de informação sobre sexualidade, seguidos dos amigos, irmãos e parentes próximos (Tabela 2). Poucos foram os relatos de busca de conhecimento com profissionais da área de saúde. Apenas uma adolescente da zona rural e duas da urbana, das 13 cuja fonte de informação sobre sexualidade foram por profissionais, relataram procurar a ajuda de um médico para elucidar esse tipo de questão, o que poderia ser explicado pela falta de profissional que atue com adolescentes no local ou talvez pelo fato de que os próprios adolescentes não saibam que podem procurar serviço médico para tirar suas dúvidas com relação à sexualidade. Além disso, muitas vezes o profissional sente dificuldades ao abordar questões pertinentes à sexualidade na adolescência ou não acredita que isso faça parte de seu trabalho ${ }^{10}$.

Cardozo et al. ${ }^{15}$, estudando o comportamento sexual de adolescentes do sexo feminino em Salvador, Bahia, relataram que a mãe é a principal interlocutora quando o assunto é sexualidade. Apesar da televisão ser também fonte de informação, as redes de televisão têm pouquíssimos programas educacionais sobre o tema. Em relação ao médico como fonte de informação, os autores tentam explicar que, possivelmente, o pouco tempo para o atendimento e as dificuldades de acesso ao profissional, principalmente na rede pública, em que 0 médico é sobrecarregado de atendimentos, fazem com que ele tenha sua atenção voltada mais para o tratamento de doenças e disponha de poucas oportunidades para abordar a prevenção.

A família é a principal reguladora da sexualidade e suas orientações são indicadoras de proibição. As informações recebidas limitam-se à explicação de regras de condutas e estão apoiadas em valores que priorizam a manutenção do sistema familiar. Os pais geralmente não percebem que a família deveria estar disponível para oferecer tais informações; assim, elas passam a ser obtidas por meio de revistas, amigas, colegas de escola, longe dos olhos dos pais ${ }^{16}$.
O modelo familiar funciona também como fator protetor para o comportamento de risco do adolescente, principalmente quando estão presentes o amor, o compromisso, o respeito e limites, com autoridade e afeto, nunca com autoritarismo, sendo necessários ensinamentos sobre o uso da liberdade vinculado à responsabilidade ${ }^{17}$.

Gomes et al. ${ }^{18}$ descreveram como insatisfatório o conhecimento dos jovens quando o assunto é sexualidade; as meninas são mais informadas que os rapazes, pois participam de forma assídua de ações sobre educação sexual.

Whitaker et al. ${ }^{19}$ observaram que a comunicação entre pais e filhos sobre o início da vida sexual e sobre sexualidade auxilia na redução do comportamento de risco e aumenta os índices de uso dos métodos preventivos durante as atividades sexuais. Da mesma maneira como escolas que tenham programas de educação sexual auxiliam nestes $\operatorname{aspectos}^{20}$.

Nos dias de hoje, é raro o adolescente não saber da existência da Aids, das DST, da camisinha, do contraceptivo oral ou de outros métodos. No entanto, observou-se que $34 \%$ das jovens da zona rural e 33\% da urbana conhecem métodos preventivos para DST e, respectivamente, 44\% e 45\% para gestação (Tabela 3). Assim, grande número de adolescentes continua tendo problemas com a sua saúde reprodutiva. Embora de modo geral não tenha havido diferença estatística entre as diversas variáveis quando se estuda o conhecimento sobre a prevenção da gravidez, o pequeno tamanho da amostra não permite conclusões satisfatórias.

Segundo Villanueva et al. ${ }^{21}$, com relação aos métodos contraceptivos, 57\% das adolescentes questionadas relataram conhecer a camisinha, 26\% os hormônios orais e 14\% o DIU.

Benvegnú et al. ${ }^{22}$ observaram estudantes do ensino médio da rede pública na cidade de Santa Maria, RS, e se depararam com os seguintes resultados: $99 \%$ responderam ser possível evitar a transmissão do vírus HIV com o uso de preservativos durante as relações sexuais; $97 \%$ com o uso de seringas descartáveis; $94 \%$ em transfusões de sangue testado para o vírus; $89 \%$ evitando o compartilhamento de seringas no uso de drogas injetáveis; $86 \%$ tomando remédios; $8 \%$ diminuindo o número de parceiros sexuais e $68 \%$ fazendo teste anti-HIV.

São várias as razões destes comportamentos sexuais desprotegidos entre adolescentes. Uma delas é a desinformação, na medida em que os adolescentes parecem desconhecer o seu período fértil ou o uso de anticoncepcionais do modo correto; ou simplesmente não acreditam na existência do risco de gravidez e doenças desde a primeira relação sexual, considerando-se indestrutíveis e inatingíveis em seu pensamento mágico.

Quando as adolescentes foram indagadas sobre o conhecimento a respeito das DST, a mais citada foi a Aids, o que mostra a eficácia das campanhas de saúde junto à população; mas as outras DST foram pouco referidas e são, muitas vezes, pouco conhecidas por grande parte dos adolescentes, como é o caso da candidíase, do HPV e do cancro mole. Esses resultados foram semelhantes aos encontrados por Chicraia et al. ${ }^{23}$, na cidade do Rio de Janeiro, RJ.

Deve-se considerar, ainda, que afirmar "conhecer uma doença" pode significar simplesmente ter ouvido falar dela e, muitas vezes, vagamente. As escolas, campanhas, serviços de saúde, enfim, todas as entidades ou pessoas envolvidas na orientação do adolescente, 
incluindo-se a sexual, devem preocupar-se não só em transmitir o conhecimento, mas em fazer reforços periódicos dos ensinamentos, pois muitas vezes os adolescentes não estão com sua atenção voltada para a questão da prevenção.

A inclusão da educação sexual nas escolas contribui para postergar a iniciação sexual e não há evidências de que 0 ensino estimule a adolescente a ter relações sexuai $5^{24,25}$, como querem alguns. Mesmo porque as DST constituem-se ainda em sério problema de saúde pública, principalmente na adolescência, podendo deixar seqüelas, curáveis ou não, como infertilidade, gravidez ectópica, câncer genital, doença hepática crônica, entre outras.

Caballero et al. ${ }^{26}$ descreveram que $40 \%$ entre todos os adolescentes que participaram de seu estudo acreditavam que a Aids podia ser transmitida por picadas de inseto, $42 \%$ em banheiros públicos e 52\% através de objetos como pratos, talheres ou copos utilizados por um portador da doença. Relataram, ainda, que o nível de conhecimento é diferente conforme o nível socieconômico e que estas falhas de conhecimento poderiam contribuir para gerar nos adolescentes crenças que os fizessem pensar que a Aids não os atingiria. No presente estudo, verificou-se a existência de percentuais mais baixos; porém, ainda se verifica a existência desse tipo de crença entre as nossos jovens, quer seja da zona rural, quer da zona urbana.

Sobre as formas de aquisição da Aids, Benvegnú et al..$^{22}$ relataram que $98 \%$ referiram relação sexual; $84 \%$ de mãe para filho durante a gravidez; $8 \%$ relação homossexual; $50 \%$ uso de lâminas de barbear; $47 \%$ relação sexual sem preservativo e $18 \%$ beijos e abraços. Responderam, ainda, não ser possível adquirir a doença através de aperto de mão (98\%); talheres (88\%); sanitários (80\%) e uso de lâminas de barbear (22\%).

Oliveira et al. ${ }^{27}$ mostraram que, de 40 adolescentes entre 14 e 20 anos de idade de uma escola pública de Fortaleza, CE, 97,5\% sabiam como se contrai a Aids. A resposta era confirmada quando 100\% deles afirmavam ter conhecimento da transmissão da doença por seringa e agulhas contaminadas e pelo sexo com pessoa portadora da doença.

\section{Conclusão}

As causas de morbi-mortalidade entre os adolescentes têm sido bastante documentadas, bem como sua associação com o comportamento pessoal. Assim, essas causas são potencialmente passíveis de prevenção. Há que se fazer um esforço cada vez maior para a união dos pais, educadores, profissionais da saúde, comunidade e mídia em um objetivo comum, qual seja a atenção integral à saúde do adolescente.

De modo geral, embora atualmente a vida sexual se inicie em idade cada vez mais precoce, os jovens não têm informações consistentes e que possam incorporar sobre o desenvolvimento e a saúde sexual e, embora recebam muitas informações sobre sexo, nem sempre sabem tanto quanto aparentam saber. Além do mais, têm pouco acesso a orientação e a serviços de planejamento familiar, sendo a fonte de seu saber, muitas vezes, conceitos equivocados, carregados de tabus, oriundos de colegas e amigos que também não tiveram acesso a educação em sexualidade. Portanto, a desinformação neste setor se torna um círculo vicioso, difícil de romper.

Apesar desta investigação não ser representativa da população de Guararema, SP, este foi o primeiro estudo realizado naquela cidade sobre características da sexualidade e do conhecimento sobre DST/ Aids e contracepção em adolescentes do gênero feminino. Este estudo aponta para a necessidade de implantaçãa de programa de educação sexual nas escolas públicas da cidade de Guararema, fazendo ainda pensar que esta poderia ser a situação encontrada em outros municípios de perfil semelhante no nosso meio, necessitando também de abordagem na esfera da sexualidade. Programas educacionais em saúde e serviços de prevenção necessitam ser implantados como ação efetiva antes que as adolescentes se envolvam em comportamentos de risco.

Conclui-se, portanto, que, apesar da maioria das adolescentes buscar informações sobre sexualidade, seus conhecimentos a respeito de doenças sexualmente transmissíveis e contracepção são inadequados tanto na zona rural como na urbana.

\section{Conflito de interesse: não há}

\section{SUMMARY}

\section{Adolescent fEMALES' KNOWLEDGE ABOUt PREGNANCY} PREVENTION METHODS AND SEXUALLY TRANSMITTED DISEASES

OBIECTIVE. To evaluate knowledge about sexuality, contraceptive methods and sexually transmitted diseases (STD) by female adolescents from both rural and urban zone attending public school.

METHODS. A cross sectional study was made with 506 teenagers, 10 to 16 years old, attending Dr. Roberto Feijó Public School in Guararema, São Paulo. A semi-structured questionnaire with general questions about sexuality, contraceptive methods and STD was administered. The Chi-square test was used to verify the association between variables.

RESULTS. Mean age of the girls from the rural zone was 13 years and I I months and from the urban zone age was 13 years and 7 months, with no statistical difference. Ofall the girls, $31 \%$ came from the rural and $69 \%$ from the urbanzone. Adolescentsfrom the ruralzonelookedformore information about sexuality $(81.2 \%)$ when compared to those from the urban zone $(72.2 \%)(p<0.0568)$. Parents were the main source of informationforboth zones. The condom was the mostfamiliarmethod in the rural (44\%)and the urban (45\%) zones $(p=0.0022)$. AIDSwas the bestknownSTD by girlsfrom the rural (43\%) and urban (39\%) zones ( $p=0.7843)$.

CONCLUSION. Most of the surveyed female adolescents sought information about sexuality, however their knowledge about STD and contraceptive methods was inadequate. [Rev Assoc Med Bras 2007; 53(I): |4-9]

KEY wORDS: Adolescent. Sexuality. Contraception. Sexually transmitted disease. Behavior.

\section{REFERÊNCIAS}

I. IBGE. Censo demográfico 2000 [on-line]. Disponível em: http://www. ibge.gov.br.

2. Guajardo SN, Õnederra RD, Henríquez MEC, Adaros KM. El adolescente y sus principales motivos de consulta en una unidade especializada. Cuad Méd Soc. (Santiago de Chile) 1983;24(2):55-9.

3. Carlini-Cotrim B, Gazal-Carvalho C, Gouveia N. Comportamento de saúde entre jovens estudantes das redes pública e privada da área metropolitana de São Paulo. Rev Saúde Pública. 2000;34(6):636-45.

4. Rickert VI, Jay MS, Gottlieb AA. Adolescent wellness. Med Clin North Am. 1990;74(5): I |35-48. 
5. Prestes RC, Colpani J, Burlamaque R, Pozza M, Zandoná JM, Herzog ML, et al. Anticoncepção e sexualidade entre escolares.Rev Med Hosp São Vicente de Paulo. 1994;6(15):22-5.

6. UNESCO. Pesquisa: juventudes e sexualidade [online]. Disponível em: http://www.observatorio.ucb.unesco.org.br/publicacoesjuventudes. [citado 2005 nov 24]

7. Albino GC, Vitalle MSS, Schussel EY, Batista N. A sexualidade pelo olhar das jovens: contribuições para a prática do médico de adolescentes. Rev Paul Pediatr. 2005;23(3): 124-9.

8. Borges ALV, Schor N. Início da vida sexual na adolescência e relações de gênero: um estudo transversal em São Paulo, Brasil, 2002. Cad Saúde Pública. 2005;2I (2):499-507.

9. Tassi MM. Adolescência [online]. Disponível em: http://www. reprodusite.hpg.ig.com.br/adolescência.htm. [citado 2003 mar]

10. Vitalle MSS. Alguns pontos conceituais sobre sexualidade na adolescência. Rev Paul Pediatr. 2003;2I(02):89-93.

II. Brasil. Ministério da Educação, 2002 [online]. Disponível em: http:// www.inpe.gov.br/donwload/censo/perfil/perfil_educ guararema_sp.csv.

12. Rodrigues Jr OM. Inventários de sexualidade: uma forma de obtenção de conhecimento complementar da vida sexual. Terapia Sexual. 1999; I(2):6I- 120.

13. Muza GM, Costa MP. Elementos para a elaboração de um projeto de promoção à saúde e desenvolvimento dos adolescentes. $\mathrm{O}$ olhar dos adolescentes. Cad Saúde Pública. 2002; | 8(I)32 |-8.

14. Martins LBM, Costa-Paiva L, D'Osis MJ, Sousa MH, Pinto Neto AM, Tadini V. Conhecimentos sobre métodos anticoncepcionais por estudantes adolescentes. Rev Saúde Pública. 2006;40( I ):57-64.

15. Cardozo DM, Freitas IC, Fontoura MSH. Comportamento sexual de adolescentes do gênero feminino de estratos sociais distintos em Salvador, Bahia, Brasil. Rev Paul Pediatr. 2002;20(3): 1 22-8.

16. Dias ACG, Gomes WB. Conversas sobre sexualidade na família e gravidez na adolescência: A percepção dos pais. Estud Psicol. (Natal) 1999;4(I):79- 106.

17. Saito MI. Adolescência, sexualidade e educação sexual. Pediatr Mod. 2001;37(1):3-6.

18. Gomes WA, Costa MCO, Sobrinho CLN, Santos CAST, Bacelar EB. Nível de informação sobre adolescência, puberdade e sexualidade entre adolescentes. J Pediatr. (Rio de J) 2002;78(4):30 I-8.
19. Whitaker DJ, Miller KS, May DC, Levin ML. Teenage partenrs' communication about sexual risk and condom use: The importance of parent-teenager discussions. Fam Plan Perpect. 1999;3 I (3): I I 7-2I.

20. Häggström-Nordin E, Hanson U, Tydén T. Sex behavior among high school students in sweden: Improvement in contraceptive use over time. J Adolesc Health. 2002;30(4):288-95.

2I. Villanueva LA, Campos R, Pérez-Fajardo MM. Conocimientos y prácticas anticonceptivas en adolescentes embarazadas. Ginec Obst Méx. 200 I;69(2):239-42

22. Benvegnú LA, Breitenbach F, Copette FR, Santos RP, Paqualotto AC, Minuzzi RS. HIV, adolescentes e sexualidade. J Bras Med. 2001;80(1):25-7.

23. Chicraia MA, Barros CRP, Cromack LMF, Meirelles ZV, Silva MRN, Baker G. Conhecimento, attitudes e práticas relacionadas à DST/AIDS: avaliação de adolescentes atendidos em uma unidade de atenção primária. DST J Bras Doenças Sex Trans 1997;9(3): I0-5.

24. Saito MI. Sex education in school: Preventing unwanted pregnancy in adolescents. International J Gynecol Obstet. 1998;63 (Suppl I): SI 57-60.

25. Hassan EA, Creatsas GC. Adolescent sexuality: A developmental milestone or risk: taking behavior? The role of health care in the prevention of sexually transmitted diseases. J Pediatr Adolesc Gynecol .2002; 13(2): 1 19-24.

26. Caballero RH, Villaseñor AS, Hidalgo ASM. Fuentes de información y su relación con el grado de conocimientos sobre el SIDA en adolescentes de México. Rev Saúde Pública. 1997;3 I (4):35 I-9.

27. Oliveira EL, Fernandes AFC, Linard AG. Conhecimento das adolescentes sobre AIDS e comportamento frente a doença. Pediatria Atual 1999; 12(10):55-63.

Artigo recebido: 23/07/04

Aceito para publicação: 23/08/06 